\title{
Desempenho de taro em função de doses de cama de aviário, sob sistema orgânico de produção
}

\author{
Fábio Luiz de Oliveira' ${ }^{1}$; José Guilherme M Guerra²; Dejair L de Almeida²; Raul de LD Ribeiro ${ }^{3}$; \\ Edmilson E da Silva ${ }^{3}$; Vinícius V Silva ${ }^{4}$; José Antonio A Espindola ${ }^{2}$ \\ ${ }^{1}$ UFVJM, Dept ${ }^{\circ}$. Agronomia, R. da Glória, 187, 39100-000 Diamantina-MG; ${ }^{2}$ Embrapa Agrobiologia, C. Postal 74505, $23890-000$ \\ Seropédica-RJ; ${ }^{3}$ UFRRJ, Dept ${ }^{\circ}$. Fitotecnia, BR 465, km 07, 23851-970 Seropédica-RJ; ${ }^{4}$ PESAGRO-RIO, Rua Euclides Solos de Pontes, \\ 30, Centro, 28625-020 Nova Friburgo-RJ; fabio.lo@ufvjm.edu.br;
}

\section{RESUMO}

O experimento foi conduzido na Região Serrana do estado do Rio de Janeiro, em Nova Friburgo, com o objetivo de avaliar o efeito da aplicação de doses de "cama" de aviário, como fonte de nitrogênio, sobre o desempenho de taro cultivado organicamente, em sistema plantio direto. O solo da área foi classificado como Cambissolo Háplico. A aveia preta (Avena strigosa) foi usada como pré-cultivo, em rotação de culturas. O delineamento experimental foi de blocos ao acaso com quatro repetições. Os tratamentos foram doses de "cama" de aviário equivalentes a 0; 50; 100 e $200 \mathrm{~kg} \mathrm{ha}^{-1}$ de N, aplicadas em cobertura por ocasião da amontoa, realizada aos 120 dias após o plantio da cultura. A adubação de cobertura com "cama" de aviário promoveu aumento significativo na produtividade de taro, que atingiu um valor máximo com a dose de $130 \mathrm{~kg} \mathrm{ha}^{-1}$ de $\mathrm{N}$. A aplicação de "cama" de aviário também favoreceu maiores teores de $\mathrm{N}$ nos rizomas de taro.

\begin{abstract}
Performance of organically grown taro in relation to poultry litter doses

An experiment was carried out in Nova Friburgo, located in the mountain region of Rio de Janeiro State, to evaluate the effect of poultry litter doses on the performance of organically grown taro, under no-tillage. The soil of the area was a Haplic Cambisol. Black oat was previously cultivated in the experimental area. The experimental design was of randomized blocks, with four replications. The treatments consisted of $0 ; 50 ; 100$ and $200 \mathrm{~kg} \mathrm{~N}^{-}$ ${ }^{1}$ of poultry litter, applied 120 days after planting. The use of poultry litter promoted a significant increase in taro yield, with a maximum value corresponding to $130 \mathrm{~kg} \mathrm{ha}^{-1}$ of $\mathrm{N}$ rate of. It also increased the $\mathrm{N}$ content of the taro rhizomes.
\end{abstract}

Keywords: Colocasia esculenta, Avena strigosa, no-tillage, poultry litter.

Palavras-chave: Colocasia esculenta, Avena strigosa, plantio direto.

(Recebido para publicação em 9 de janeiro de 2007; aceito em 25 de abril de 2008)

$\mathrm{O}$ taro (Colocasia esculenta), também conhecido como inhame, é uma olerícola da família Araceae, com considerável valor nutricional, apresentando elevado teor de amido, o que permite tanto seu consumo in natura quanto sua utilização como matéria-prima para a agroindústria (Vilpoux, 2001). A sua parte comestível é formada por um rizoma central esférico, rodeado por vários rebentos laterais, sendo que esses últimos geralmente obtêm maior valor de comercialização (Puiatti, 2002).

Dentre as estratégias que caracterizam o manejo da fertilidade do solo nos sistemas orgânicos de produção, destaca-se a aplicação de adubos orgânicos. De maneira geral, essa prática favorece a manutenção da matéria orgânica do solo, melhorando suas propriedades físicas, químicas e biológicas. Do ponto de vista físico, o uso de estercos promove o aumento da estabilidade de agregados, associado à redução da densidade do solo (Andreola et al., 2000). Com relação às propriedades químicas, des- taca-se o aumento da disponibilidade de nutrientes para as culturas e da capacidade de troca de cátions (CTC), associados à complexação de elementos tóxicos (Bayer \& Mielniczuk, 1999). A matéria orgânica também auxilia a atividade dos organismos do solo, o que por sua vez resulta em impactos positivos sobre a ciclagem de nutrientes (Filser, 1995).

Dentre os adubos orgânicos empregados na olericultura, merece destaque a "cama" de aviário, que normalmente apresenta elevados teores de nutrientes. Oliveira (2001) observou teores de nitrogênio $\left(33,6 \mathrm{~g} \mathrm{~kg}^{-1}\right)$, fósforo $(13,3 \mathrm{~g}$ $\left.\mathrm{kg}^{-1}\right)$, potássio $\left(19,2 \mathrm{~g} \mathrm{~kg}^{-1}\right)$ e cálcio $(25,5$ $\mathrm{g} \mathrm{kg}^{-1}$ ), superiores a outra fonte de adubo orgânico muito usada, o esterco de gado, que apresentou os teores: nitrogênio $\left(15,8 \mathrm{~g} \mathrm{~kg}^{-1}\right)$, fósforo $\left(1,9 \mathrm{~g} \mathrm{~kg}^{-1}\right)$, potássio $\left(13,2 \mathrm{~g} \mathrm{~kg}^{-1}\right)$ e cálcio $\left(18 \mathrm{~g} \mathrm{~kg}^{-1}\right)$. A "cama" de aviário tem apresentado resultados positivos para a produção de diversas hortaliças como repolho (Oliveira et al., 2003), brócolis (Moreira,
2003), taro (Zarate et al., 2000) e milho (Zarate et al., 2003).

A adubação orgânica pode ter sua eficiência aumentada em sistemas conservacionistas de preparo do solo, como o plantio direto. Particularmente para os agricultores familiares, são necessários esforços para o desenvolvimento de sistemas alternativos, havendo uma forte demanda no que diz respeito ao cultivo de hortaliças como o taro (Oliveira, 2004).

O objetivo do presente trabalho foi avaliar os efeitos da aplicação de doses crescentes de "cama" de aviário sobre o desempenho produtivo do taro, em sistema orgânico de produção, sob plantio direto, nas condições edafoclimáticas da região Serrana Fluminense.

\section{MATERIAL E MÉTODOS}

O experimento foi instalado na Estação Experimental de Nova Friburgo (EENF) da PESAGRO-RIO, localizada 


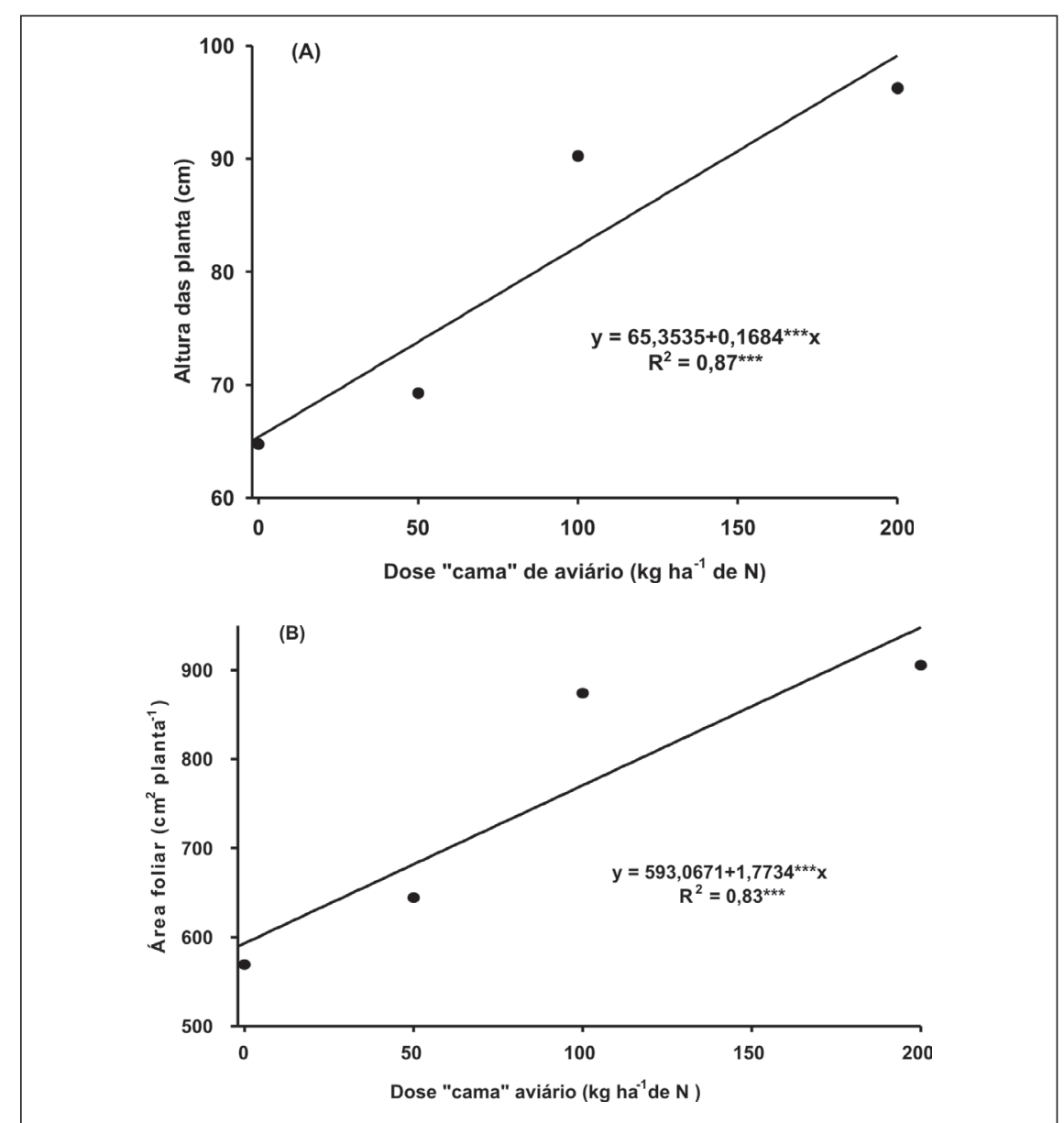

Figura 1. Altura (A) e área foliar (B) das plantas de taro aos 210 dias após o plantio em função de doses de "cama" de aviário em cobertura (height (A) and leaf area (B) of taro plants, 210 days after planting date depending on poultry litter doses in covering). Nova Friburgo, UFRRJ, 2004.

na região Serrana Fluminense, em 2002. A Estação Experimental fica a 1010 m de altitude, em região de temperaturas amenas (média anual de $18^{\circ} \mathrm{C}$ ) e precipitação anual de $1300 \mathrm{~mm}$.

O solo da área experimental foi classificado como Cambissolo Háplico, sendo anteriormente cultivado com hortaliças. A análise de solo, na profundidade de $0-20 \mathrm{~cm}$, apresentou: $\mathrm{pH}$ em água $=5,6 ; \mathrm{Al}=0,0 \mathrm{cmol}_{\mathrm{c}} \mathrm{dm}^{-3} ; \mathrm{Ca}=4,3$ $\mathrm{cmol}_{\mathrm{c}} \mathrm{dm}^{-3} ; \mathrm{Mg}=1,3 \mathrm{cmol}_{\mathrm{c}} \mathrm{dm}^{-3} ; \mathrm{K}=$ $0,52 \mathrm{cmol}_{\mathrm{c}} \mathrm{dm}^{-3}$ e $\mathrm{P}=63 \mathrm{mg} \mathrm{kg}^{-1}$. Não foi feita adubação de plantio, procedendo-se a adubação de cobertura com aplicação das doses estipuladas para cada tratamento.

O delineamento experimental foi de blocos ao acaso, com quatro repetições. Os tratamentos consistiram nas doses de "cama" de aviário equivalentes a 0; 1,69 $\mathrm{t} \mathrm{ha}^{-1}\left(50 \mathrm{~kg} \mathrm{ha}^{-1}\right.$ de N); 3,38 t ha-1 (100 $\mathrm{kg} \mathrm{ha}^{-1}$ de N$)$ e $6,75 \mathrm{tha}^{-1}\left(200 \mathrm{~kg} \mathrm{ha}^{-1} \mathrm{de}\right.$ $\mathrm{N}$ ), aplicadas em cobertura por ocasião da operação de amontoa, realizada aos 120 dias após o plantio da cultura. As parcelas comportaram 120 plantas de taro, no espaçamento de $1,0 \times 0,3 \mathrm{~m}$, dispostas em quatro linhas de plantio, considerando-se como área útil aquela ocupada por cinco plantas centrais em cada uma das duas linhas centrais de cada parcela.

A aveia preta foi semeada a lanço, no mês de maio, como adubo verde na forma de pré-cultivo. Realizou-se seu corte aos 120 dias após a semeadura, sendo a palhada mantida em cobertura do solo para todos os tratamentos. Os rizomas-semente de taro utilizados representam um ecotipo preferido pelos produtores da região, apresentando bainha foliar de cor verde e rizomas de polpa branca.
As análises da composição química da "cama" de aviário, bem como os teores de $\mathrm{N}$ contidos na biomassa da aveia preta foram determinados de acordo com o método recomendado por Bremner \& Mulvaney (1982), enquanto $\mathrm{P}, \mathrm{K}, \mathrm{Ca}$ e $\mathrm{Mg}$ foram determinados a partir da digestão nítrico-perclórica (Bataglia et al., 1983). A determinação de $\mathrm{P}$ foi feita por colorimetria através da formação da cor azul do complexo fosfato-molibdato em presença de ácido ascórbico, e de $\mathrm{K}$ por espectrofotometria de absorção atômica (Embrapa, 1997). Por sua vez, as determinações de $\mathrm{Ca}$ e de $\mathrm{Mg}$ foram feitas por espectrofotometria de absorção atômica (Bataglia et al., 1983).

As quantidades de nutrientes contidos na "cama" de aviário com base na matéria seca foram $29,6 \mathrm{~g} \mathrm{~kg} \mathrm{~g}^{-1} \mathrm{de} \mathrm{N}, 26,6$ $\mathrm{g} \mathrm{kg}^{-1} \mathrm{P}, 38,0 \mathrm{~g} \mathrm{~kg}^{-1}$ de K, $47 \mathrm{~g} \mathrm{~kg}^{-1}$ de Ca e 6,6 $\mathrm{g} \mathrm{kg}^{-1} \mathrm{de} \mathrm{Mg}$.

As características fitotécnicas do taro avaliadas aos 210 dias após o plantio, foram: (1) altura da planta (tomada do nível do solo até a inserção do pecíolo no limbo da folha mais alta) e (2) área foliar por planta (obtida pelo somatório da área de cada folha da planta). Em cada folha, a área foi calculada pela mensuração das três diagonais ligando o ponto de inserção do pecíolo aos três vértices do limbo, segundo o método de Nolasco (1984). Os componentes de produção avaliados foram: (1) número de rebentos por planta, (2) produtividade de rebentos e (3) produtividade de rizoma central. Além disso, avaliou-se o teor de nitrogênio das folhas e dos rebentos (Bremner \& Mulvaney, 1982).

Os dados foram submetidos à análise de variância, através do teste $\mathrm{F}$, e análise de regressão, de acordo com Pimentel-Gomes (1990).

\section{RESULTADOS E DISCUSSÃO}

A produção de biomassa e as quantidades de nutrientes acumulados na palhada de aveia preta foram de 9,1 t ha $^{-1}$ de matéria seca, contendo de 130 $\mathrm{kg} \mathrm{ha}^{-1}$ de N, $14 \mathrm{~kg} \mathrm{ha}^{-1}$ de P, $174 \mathrm{~kg} \mathrm{ha}^{-1}$ de K, $33 \mathrm{~kg} \mathrm{ha}^{-1}$ de Ca e $21 \mathrm{~kg} \mathrm{ha}^{-1}$ de $\mathrm{Mg}$.

A aplicação de doses crescentes de "cama" de aviário proporcionou aumen- 
tos lineares na altura e área foliar das plantas (Figura 1). De forma similar, Zarate et al. (2000) observaram maiores valores de matéria fresca de limbos e pecíolos de taro, por ocasião da colheita, em plantas adubadas com "cama" de frango de corte semi-decomposta. Esses resultados podem ser associados ao suprimento de nutrientes, principalmente nitrogênio, por aquele tipo de adubo orgânico e pelos resíduos da aveia preta. De acordo com Jacobs \& Clark (1993), o maior fornecimento de N para plantas de taro favorece o desenvolvimento de sua parte aérea (folhas e pecíolos), atuando como fator determinante para a produção de biomassa. Isso está associado à ausência de uma área foliar ótima no taro durante grande parte de seu ciclo (Goenaga, 1995).

Deve-se ressaltar que, de acordo com Jansson (1978), há uma correlação positiva entre o suprimento de nutrientes, principalmente $\mathrm{N}$ e K, e a duração do ciclo da cultura do taro. Segundo esse autor, ocorre maior produção de biomassa de parte aérea quando o suprimento é feito entre o terceiro e o quinto mês, pois os nutrientes estariam sendo fornecidos em época de maior requerimento pelas plantas. Os resultados apresentados reforçam essa afirmativa, pois a adubação em cobertura, realizada aos 120 dias, significou maior desenvolvimento da parte aérea das plantas (Figura 1).

Observou-se efeitos quadráticos significativos da aplicação de doses de "cama" de aviário sobre o número de rebentos por planta (Figura 2). Esse resultado respalda os valores obtidos por Puiatti (1987), que destacou a influência do fornecimento de uma fonte orgânica com elevado teor de $\mathrm{N}$ sobre os ganhos em quantidade de rebentos laterais.

Efeitos quadráticos significativos da aplicação de doses de "cama" de aviário também foram observados sobre as produtividades de rebentos e de rizoma central (Figura 3). Com base nas equações de regressão obtidas, foi possível estimar a dose de máxima produtividade de rebentos, que se situou em torno de $130 \mathrm{~kg} \mathrm{ha}^{-1}$ de $\mathrm{N}$ (aproximadamente 4,4 t ha-1 de "cama" de aviário).

Outros trabalhos mostraram valores próximos do obtido para a dose de má-

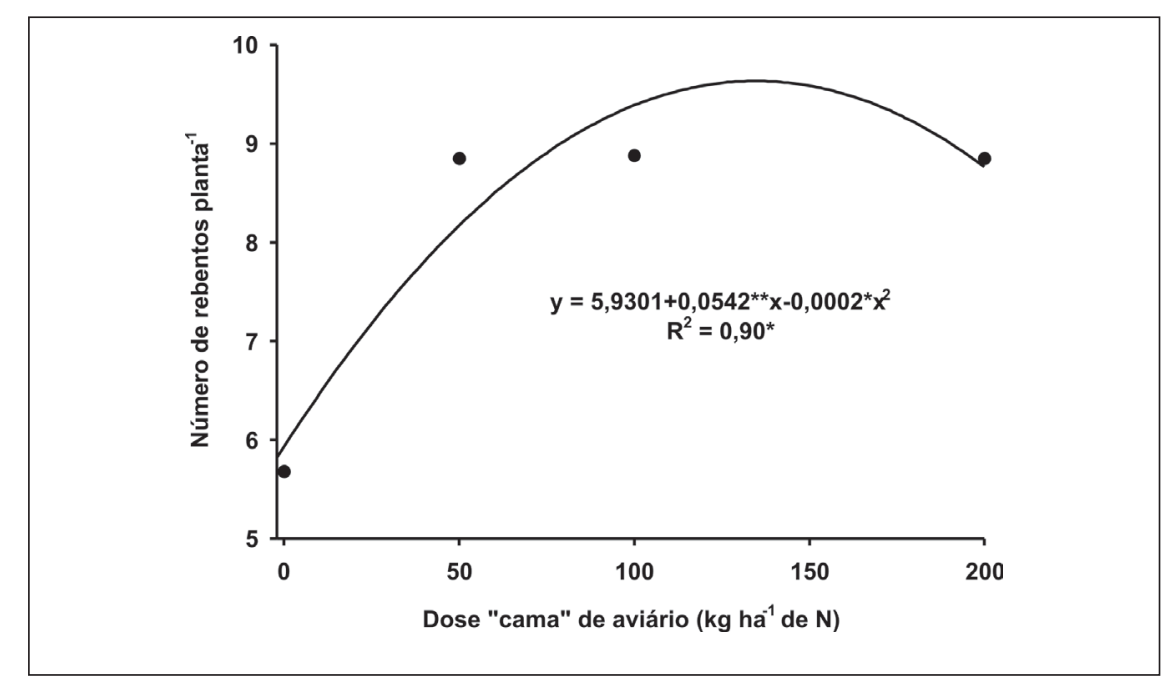

Figura 2. Número de rebentos de taro em função de doses de "cama" de aviário em cobertura (number of taro tillers depending on poultry litter doses in covering). Nova Friburgo, UFRRJ, 2004.

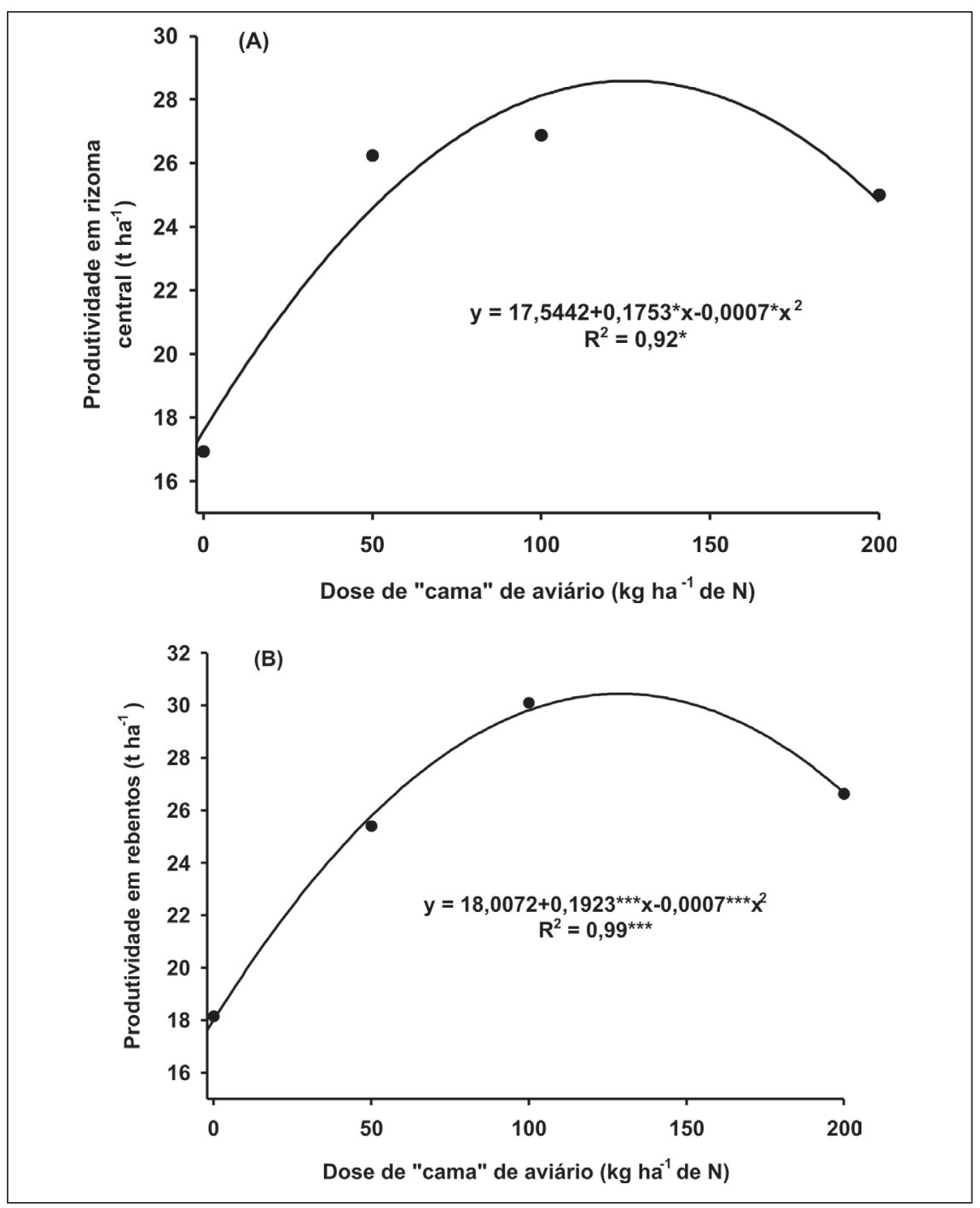

Figura 3. Produtividade de rizomas centrais (A) e rebentos (B) de taro em função de doses crescentes de "cama" de aviário em cobertura (yield of central rhizomes (A) and tillers (B) of taro depending on poultry litter doses in covering). Nova Friburgo, UFRRJ, 2004. 

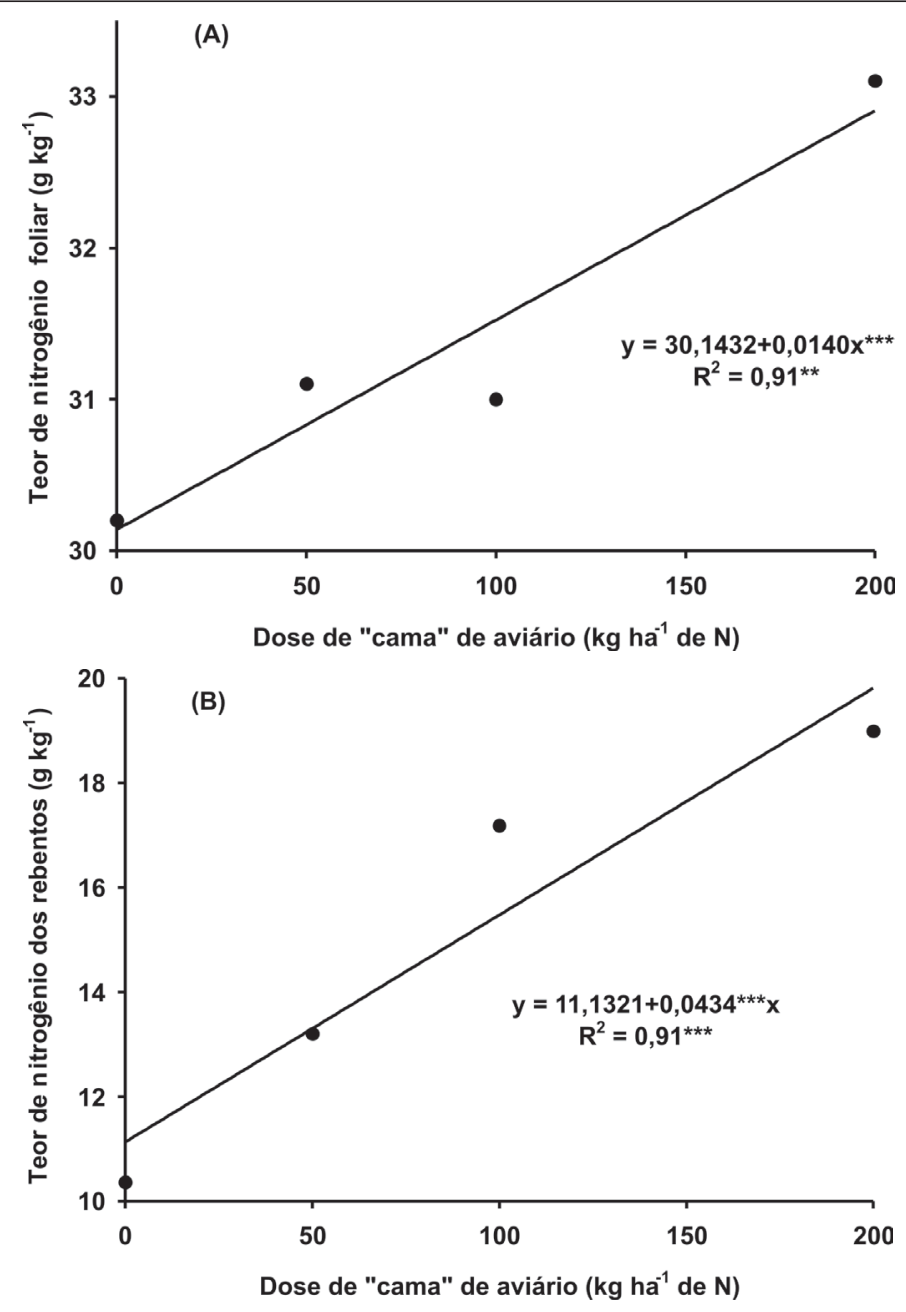

Figura 4. Teor de nitrogênio em folhas (A) e rebentos (B) do taro na colheita, em função de doses de "cama" de aviário em cobertura (Nitrogen content in leaves (A) and tillers (B) of taro depending on poultry litter doses in covering). Nova Friburgo, UFRRJ, 2004.

xima produtividade na adubação orgânica do taro. Almeida et al. (1986) obtiveram maior produtividade quando se utilizaram $30 \mathrm{t} \mathrm{ha}^{-1}$ de esterco de curral, significando o aporte de, aproximadamente, $180 \mathrm{~kg} \mathrm{ha}^{-1} \mathrm{de} \mathrm{N}$. Por sua vez, Pimenta (1993) encontrou maior produção de rebentos comercializáveis quando se aplicou, aos 110 dias após o plantio de taro, uma dose equivalente a 200 $\mathrm{kg} \mathrm{ha}^{-1} \mathrm{de} \mathrm{N}$, como composto formado a partir de capim gordura (Melinis minutiflora) e esterco de curral, demonstrando que a dose é dependente do tipo de solo, fonte de nitrogênio utilizada e manejo adotado.

A aplicação de "cama" de aviário proporcionou ainda aumento linear nos teores de nitrogênio das folhas e rebentos de taro (Figura 4). Resultados semelhantes foram descritos por Jansson (1978), que observou que a aplicação de nitrogênio induzia a aumentos na produtividade e no teor de $\mathrm{N}$ nos rebentos. Esse comportamento pode ser atribuído ao fornecimento de N pela "cama" de aviário, sendo que para a dose de máxima produtividade no presente trabalho $\left(130 \mathrm{~kg} \mathrm{ha}^{-1} \mathrm{de} \mathrm{N}\right)$, obteve-se um teor de nitrogênio de $16,7 \mathrm{~g}$ $\mathrm{kg}^{-1}$ de $\mathrm{N}$ nos rebentos. Considerando-se que parte desse nutriente encontra-se na forma de proteína no taro, a prática da adubação orgânica com "cama" de aviário favoreceu o aumento da qualidade nutricional dessa hortaliça.

A adição de $4,4 \mathrm{t} \mathrm{ha}^{-1}$ de "cama" de aviário (equivalente a $130 \mathrm{~kg} \mathrm{ha}^{-1} \mathrm{de} \mathrm{N}$ ) possibilitou maior desenvolvimento vegetativo das plantas, associado a uma elevada produtividade.

\section{REFERÊNCIAS}

ALMEIDA DL; VASCONCELLOS HO; SAKE RC; PESSANHA GG. 1986. Efeito do espaçamento e da adubação orgânica na produção de taro (Colocasia esculenta). Itaguaí: PESAGRO-RIO/EEI. 3 p. (Comunicado Técnico, 157).

ANDREOLA F; COSTA LM; OLSZEVSKI N. 2000. Influência da cobertura vegetal de inverno e da adubação orgânica e, ou, mineral sobre as propriedades físicas de uma Terra Roxa Estruturada. Revista Brasileira de Ciência do Solo 24: 857-865.

BATAGLIAOC; FURLANI AMC; TEIXEIRAJPF; GALLO JR. 1983. Métodos de análise química de plantas. Campinas: IAC. n.p. (Instituto Agronômico de Campinas. Boletim, 78).

BAYER C; MIELNICZUK J. 1999. Dinâmica e função da matéria orgânica. In: SANTOS GA; CAMARGO FAO. (eds.). Fundamentos da matéria orgânica do solo: ecossistemas tropicais e subtropicais. Porto Alegre: Genesis. p. 9-26.

BREMNER JM; MULVANEY CS. 1982. Nitrogen total. In: PAGE AL. (ed.). Methods of soil analysis. Part 2. 2. ed. Madison: SSSA. p. $595-624$.

EMBRAPA - Empresa Brasileira de Pesquisa Agropecuária. Centro Nacional de Pesquisa de Solos. 1997. Manual de métodos de análise de solo. Rio de Janeiro. 212 p.

FILSER J. 1995. The effect of green manure on the distribution of collembola in a permanent row crop. Biology and Fertility of Soils 19: 303-308.

GOENAGA R. 1995. Accumulation and partitioning of dry matter in taro [Colocasia esculenta (L.) Schott]. Annals of Botany 76: 337-341.

JACOBS BC; CLARKE J. 1993. Accumulation and partitioning of dry matter and nitrogen in traditional and improved cultivars of taro (Colocasia esculenta (L.) Schott) under varying nitrogen supply. Field Crops Research 31: 317-328.

JANSSON SL. 1978. Potassium requirements of root crops. In: CONGRESS OF THE INTERNATIONAL POTASH INSTITUTE, 11., Bern. Proceedings... Bern: International Potash Institute, p. 276-282.

MOREIRA VF. 2003. Comportamento de duas cultivares de brócolis ramoso em aléias de feijão guandu e com níveis crescentes de esterco de aves. Rio de Janeiro: UFRRJ. 80p. (Tese mestrado).

NOLASCO F. 1984. Estudos para o cultivo inundado de taro (Colocasia esculenta) em monocultivo e em consórcio com Azolla. Viçosa: UFV. 80p. (Tese mestrado).

OLIVEIRA, FL. 2001. Manejo orgânico da cultura do repolho (Brassica oleracea var. capitata): adubação orgânica, adubação verde e consorciação. Rio de Janeiro: UFRRJ. 87p. (Tese mestrado).

OLIVEIRA FL; RIBEIRO RLD; SILVA VV; GUERRA JGM; ALMEIDA DL. 2003. Uso do pré-cultivo de Crotalaria juncea e de doses crescentes de "cama" de aviário na produção do repolho sob manejo orgânico. Agronomia 37: 60-66. 
OLIVEIRA FL. 2004. Alternativas para o manejo orgânico do taro (Colocasia esculenta $L$. Schott) em diferentes condições edafoclimáticas no estado do Rio de Janeiro. Rio de Janeiro: UFRRJ. 96 p. (Tese doutorado).

OLIVEIRA FL; RIBEIRO RLD; SILVA VV; GUERRA JGM; ALMEIDA DL. 2004. Desempenho do taro (taro) em plantio direto e no consórcio com crotalária, sob manejo orgânico. Horticultura Brasileira 22: 638-641.

PIMENTA DS. 1993. Crescimento e produção de taro (Colocasia esculenta), com composto orgânico, amontoa e capina. Viçosa: UFV. 78 p. (Tese mestrado).

PIMENTEL-GOMES F. 1990. Curso de estatística experimental. 13. ed. Piracicaba: Nobel. 234 p.
PUIATTI M. 1987. Efeito dos resíduos vegetais, bagaço de cana-de-açucar e capim gordura, $e$ do nitrogênio sobre a cultura do taro (Colocasia esculenta) cv. "Chinês". 1987. Viçosa: UFV. 75 p. (Tese mestrado).

PUIATTI M. 2002. Manejo da cultura do taro In: CARMO CAS (ed.). Taro e taro: sistemas de produçãofamiliar. Vitória: INCAPER. p. 203-252.

PUIATTI M; GREEMAN S; KATSUMOTO R; FAVERO C. 1992. Crescimento e absorção de macronutrientes pelo taro 'Chinês' e 'Japonês' Horticultura Brasileira 10: 89-92.

SANTI A; AMADO TJC; ACOSTA JAA. 2003. Adubação nitrogenada na aveia preta. I. Influência na produção de matéria seca e ciclagem de nutrientes sob sistema de plantio direto. Revista Brasileira de Ciência do Solo 27: $1075-1083$.
VILPOUX O. 2001. Produção e uso de amido. In: CEREDA MP. (ed.) Propriedades gerais do amido. São Paulo: Fundação Cargill. p. 7 28. (Série Tuberosas Amiláceas Latino Americanas, v. 1).

ZARATE NAH; VIEIRA MC; ROSA JUNIOR EJ; SILVA CG. 2000. Formas de adição de cama-de-frango de corte semidecomposta para a produção de taro. Horticultura Brasileira 18: 521-523.

ZARATE NAH; VIEIRA MC. 2003. Produção de milho doce em sucessão ao plantio de diferentes cultivares de inhame e adição de cama-de-aviário. Horticultura Brasileira 21: 5-10. 